\title{
Protein Conformational Change is Essential For Reductive Activation of Lytic Polysaccharide
}

\section{Monooxygenase by Cellobiose Dehydrogenase}

Erik Breslmayr ${ }^{1,2,3}$, Christophe V.F.P. Laurent ${ }^{2,3}$, Stefan Scheiblbrandner ${ }^{2}$, Anita Jerkovic ${ }^{2}$, Derren J. Heyes $^{1}$, Chris Oostenbrink ${ }^{3}$, Roland Ludwig' ${ }^{2}$,Tobias M. Hedison ${ }^{1,4^{*}}$, Nigel S. Scrutton ${ }^{1,4}$, Daniel Kracher $^{1,2 *}$

${ }^{1}$ Manchester Institute of Biotechnology, The University of Manchester, M1 7DN Manchester, U.K.

${ }^{2}$ Biocatalysis and Biosensing Laboratory, Department of Food Science and Technology University of Natural Resources and Life Sciences, Muthgasse 18, 1190 Vienna, Austria

${ }^{3}$ Institute of Molecular Modeling and Simulation, Department of Material Sciences and Process Engineering, Muthgasse 18, 1190 Vienna, Austria

${ }^{4}$ EPSRC/BBSRC funded Future Biomanufacturing Research Hub, The Manchester Institute of Biotechnology, The University of Manchester, M1 7DN Manchester, U.K.

*To whom correspondence should be addressed.

Email: danielkracher@boku.ac.at and tobias.hedison@manchester.ac.uk

Keywords: protein dynamics, interdomain electron transfer, interprotein electron transfer, cellobiose dehydrogenase, domain movement, lytic polysaccharide monooxygenase. 
Table S1. Solvent accessible areas and water surface densities of CDH from $C$. hotsonii.

Structure-guided homology models were generated using SWISS-MODEL (http://swissmodel.expasy.org). Solvent accessible surface areas were calculated with PyMOL (Schrödinger, LLC) and calculation of protein hydration is based on an estimate of $0.0775 \mathrm{H}_{2} \mathrm{O}$ molecules per $\AA^{2}{ }^{1}$

\begin{tabular}{llcccc}
\hline Structure & \multicolumn{1}{c}{ pdb code } & $\begin{array}{c}\text { Solvent accessible } \\
\text { surface area }\left(\AA^{2}\right)\end{array}$ & $\begin{array}{c}\text { open - } \\
\text { closed }\left(\Delta \AA^{2}\right)\end{array}$ & $\begin{array}{c}\text { structure-bound } \\
\mathbf{H}_{2} \mathbf{O} \\
\text { molecules }\end{array}$ & $\begin{array}{c}\text { open - closed } \\
\left(\mathbf{H}_{2} \mathbf{O}\right. \\
\text { molecules })\end{array}$ \\
\hline $\begin{array}{l}\text { C. } \text { hotsonii } \\
\text { closed }\end{array}$ & 4QI6 & & & & - \\
open & modelled on 4QI7, chain A & 31439.6 & 1961.7 & 2285 & 152 \\
open & modelled on 4QI7, chain B & 31252.7 & 1774.9 & 2437 & 138 \\
\hline
\end{tabular}




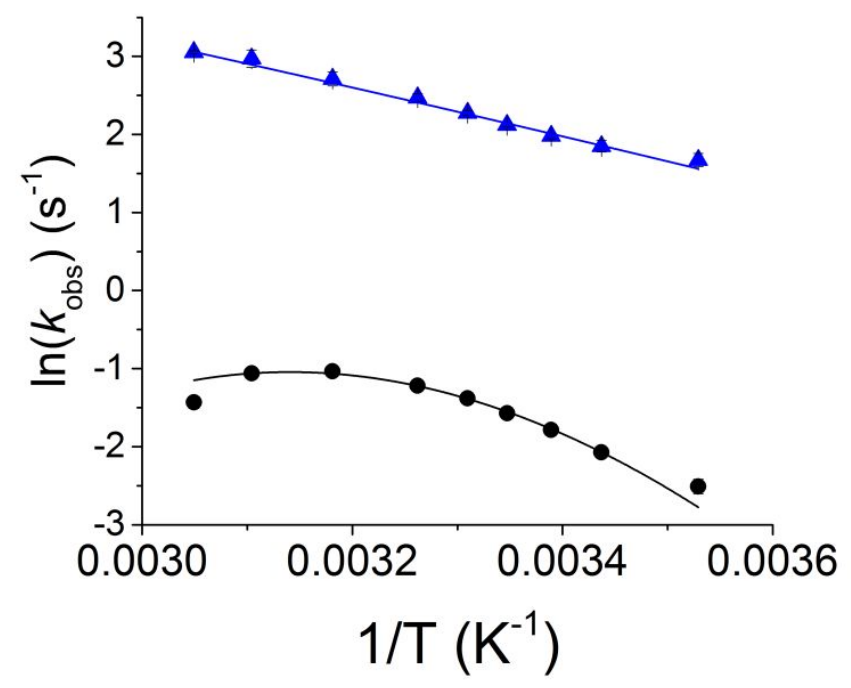

Figure S1. Arrhenius plots of the FAD reduction ( $k_{1}$, blue triangles) and the intraprotein electron transfer reaction ( $k_{2}$, black circles) and corresponding non-linear Eyring fits (Eq. S1). Thermodynamic parameters derived from fits to the non-linear Erying function $\left(\Delta H_{\mathrm{T} 0}{ }^{\ddagger}\right.$, $\Delta S_{\mathrm{T} 0}^{\ddagger}$ and $\Delta C_{\mathrm{p}}^{\ddagger}$ ) are shown in Table 1. Observed electron transfer reactions of CDH were modelled by absolute rate theory, best described by the non-linear Eyring function (Eq. S1). A linear correlation between the observed rates and temperature with a corresponding $\Delta C_{\mathrm{p}}{ }^{\ddagger}$ value of $-0.05 \mathrm{~kJ} \mathrm{~mol}^{-1} \mathrm{~K}^{-1}$ are seen for FAD reduction. In contrast, the intraprotein electron transfer rates notably deviated from linearity in the Arrhenius plot, with a negative $\Delta C_{\mathrm{p}}{ }^{\ddagger}$ value of -2.04 $\pm 0.17 \mathrm{~kJ} \mathrm{~mol}^{-1} \mathrm{~K}^{-1}$ determined from our analysis. The observed decline of the heme $b$ reduction rate above $310 \mathrm{~K}$ cannot be explained by the formation of noncatalytic partially unfolded states (temperature-dependent steady-state assays conducted over $300 \mathrm{~s}$ with cytochrome $c$ as CYTselective electron donor showed maximal activity at a temperature of $330 \mathrm{~K}$ ).

$$
\ln k_{o b s}=\ln \left(\frac{k_{B} T}{h}\right)-\Delta H_{T_{0}}^{\ddagger}+\Delta C_{p}^{\ddagger}
$$

where $\Delta H_{\mathrm{T} 0}{ }^{\ddagger}$ and $\Delta S_{\mathrm{T} 0}{ }^{\ddagger}$ denote the difference in enthalpy and entropy, respectively, at a reference temperature, $T_{0}$, of $303.15 \mathrm{~K} . \Delta C_{\mathrm{p}}{ }^{\ddagger}$ is the difference in heat capacity between the reactant and transition states, and $k_{\mathrm{B}}, h$, and $R$ are the Boltzmann, Planck, and ideal gas constants, respectively 

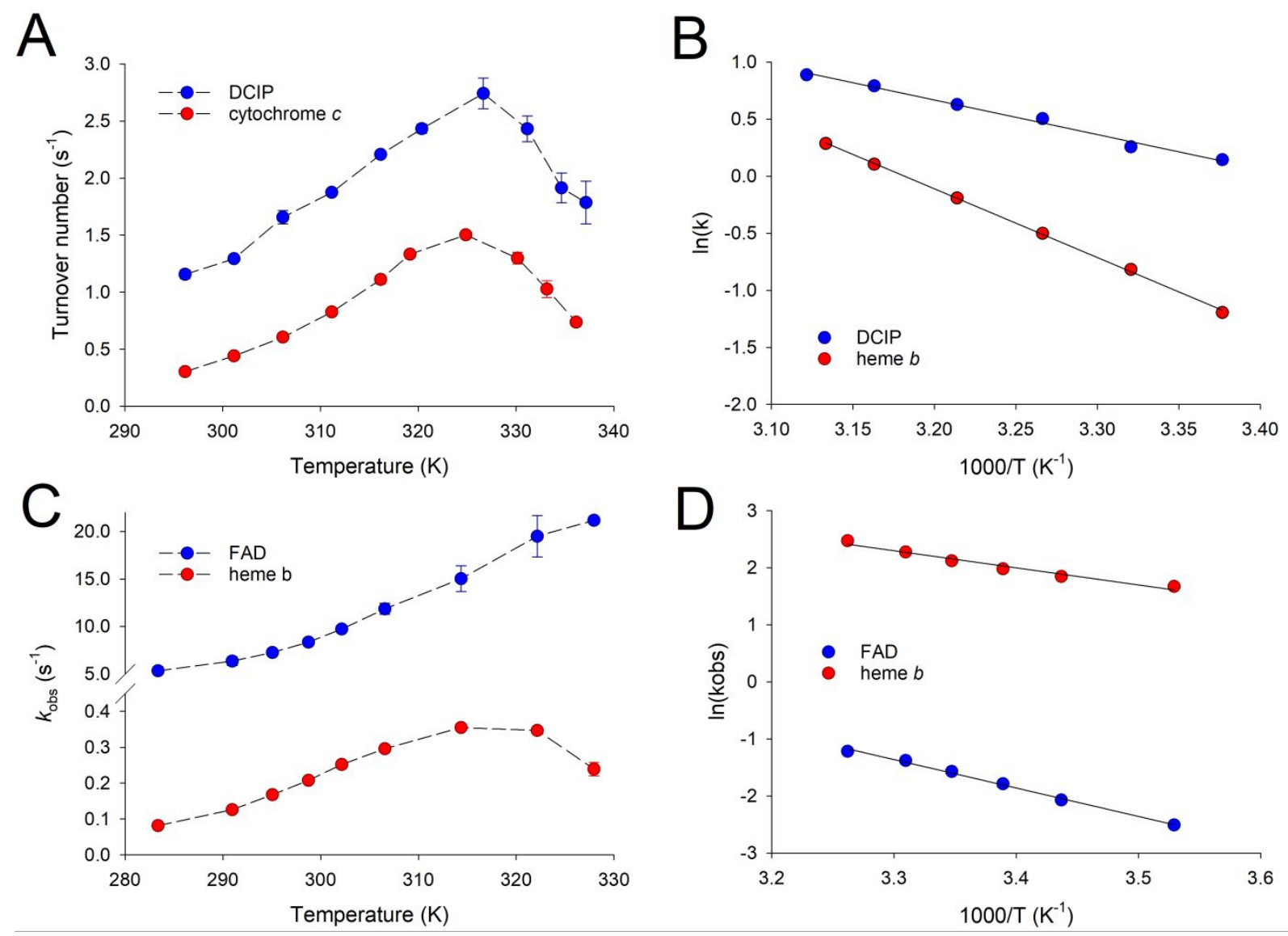

Figure S2. Temperature-dependence of $\mathrm{CDH}$-catalyzed electron transfer reactions and reduction of artificial electron acceptors. $\boldsymbol{A}$, temperature dependence of the CDH-catalyzed reduction of the FAD-dependent electron acceptor dichloroindophenol (DCIP, blue circles) and the heme $b$-dependent electron acceptor cytochrome $c$ (red circles). Reaction mixtures contained $10 \mathrm{mM}$ cellobiose and $300 \mu \mathrm{M}$ DCIP or $20 \mu \mathrm{M}$ cytochrome $c$ and were performed at $30^{\circ} \mathrm{C}$ in $50 \mathrm{mM}$ sodium acetate buffer, $\mathrm{pH}$ 4.5. Error bars show \pm S.D. $(\mathrm{n}=3)$. $\boldsymbol{B}$, Fits of the linear range of the temperature profile to the Arrhenius equation. Indicated are the activation energies obtained from the slope of the linear fits. $\boldsymbol{C}$, temperature-dependent stopped-flow traces of the FAD reduction (blue) circles and the heme $b$ reduction shown in Figure S1. Data were recorded as described in Figure S1. $\boldsymbol{D}$, calculation of the activation energies by fitting the linear range shown in Figure S2A to the Arrhenius equation. 

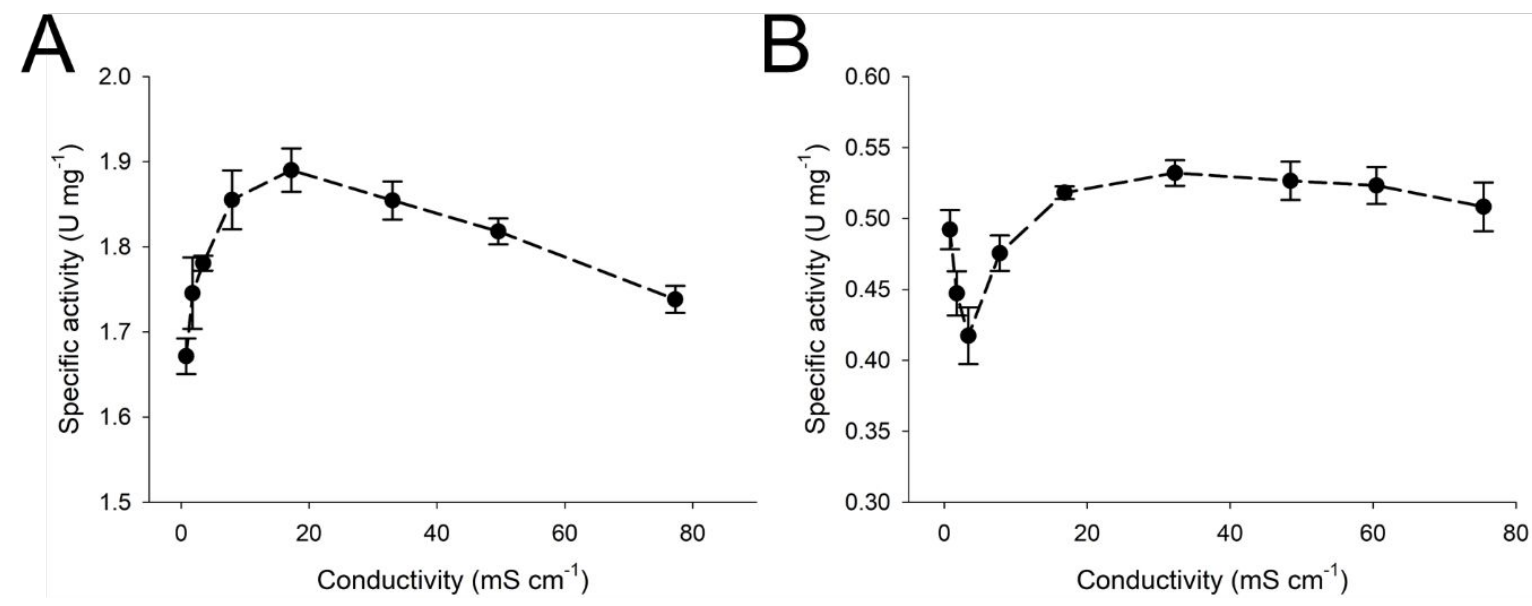

Figure S3. Effect of the solvent conductivity on the steady-state turnover of CDH. $A$, reduction of the FAD-dependent electron acceptor dichloroindophenol (DCIP); $\boldsymbol{B}$, reduction of the heme $b$-specific electron acceptor cytochrome $c$. All assays were carried out at $30^{\circ} \mathrm{C}$ and $\mathrm{pH} 4.5$ in $50 \mathrm{mM}$ sodium acetate buffer. Reactions contained $10 \mathrm{mM}$ cellobiose and $300 \mu \mathrm{M}$ DCIP, or $20 \mu \mathrm{M}$ cytochrome $c$. Error bars show \pm S.D. $(n=3)$. 
[the full-length sequence alignment is attached to the manuscript as separate supporting file]

Figure S4. Multiple sequence alignment of CDH. 362 putative sequences of ascomycetous and basidiomycetous CDHs were aligned using the structure-based MAFFT-DASH algorithm. The corresponding phylogenetic tree indicating three distinct CDH classes (class I, blue; class II, red; class III, green) is shown next to it. 

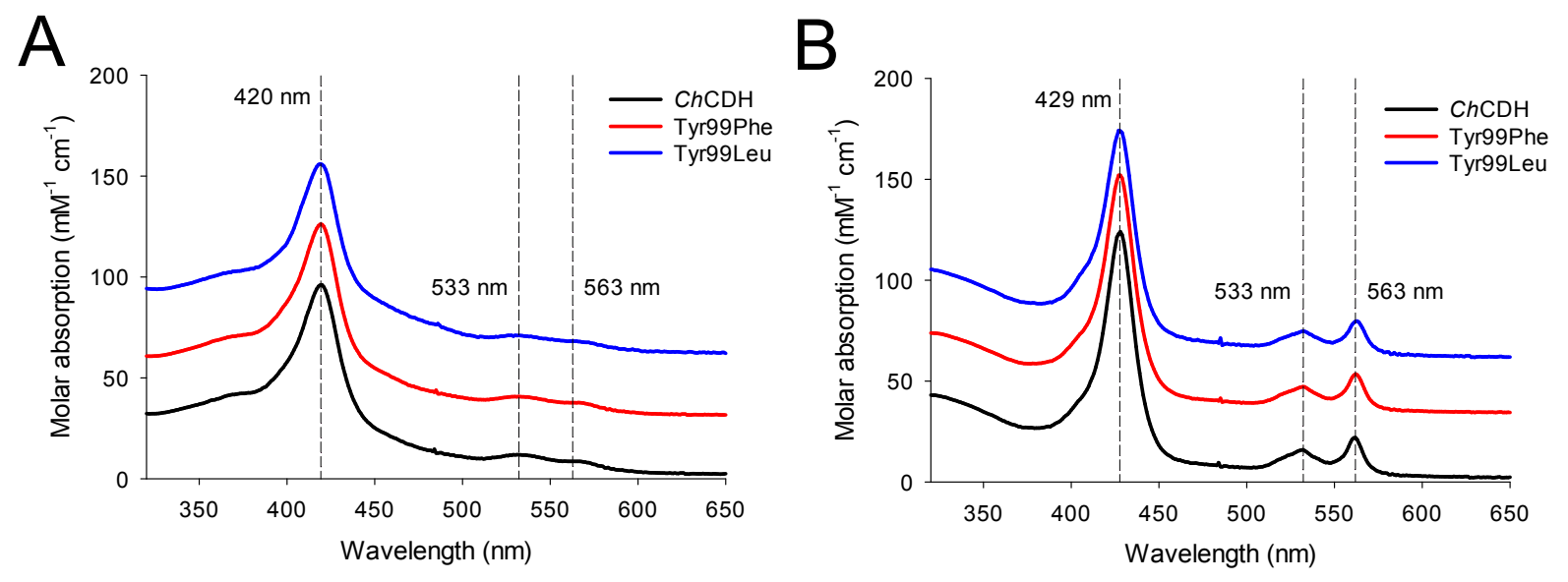

Figure S5. UV-Vis spectra of ChCDH (black), Tyr99Phe (red) and Tyr99Leu (blue). A,

CDHs in their oxidized state show the typical absorbance of the Soret band with a maximum at $420 \mathrm{~nm}$ and the weak $\alpha$ - and $\beta$-bands at $533 \mathrm{~nm}$ and $563 \mathrm{~nm}$, respectively. $\boldsymbol{B}$, spectra of reduced $\mathrm{CDH}$ variants containing $2 \mathrm{mM}$ cellobiose (ca. 1000-fold molar excess over enzymes). Reduction leads to a shift in the Soret band to $429 \mathrm{~nm}$, and to an increase in the absorbance of the heme $\alpha$ - and $\beta$-bands. For a better comparison, all spectra are vertically shifted. Spectra were recorded at $23^{\circ} \mathrm{C}$ in $50 \mathrm{mM}$ sodium acetate buffer, $\mathrm{pH} 4.5$. 

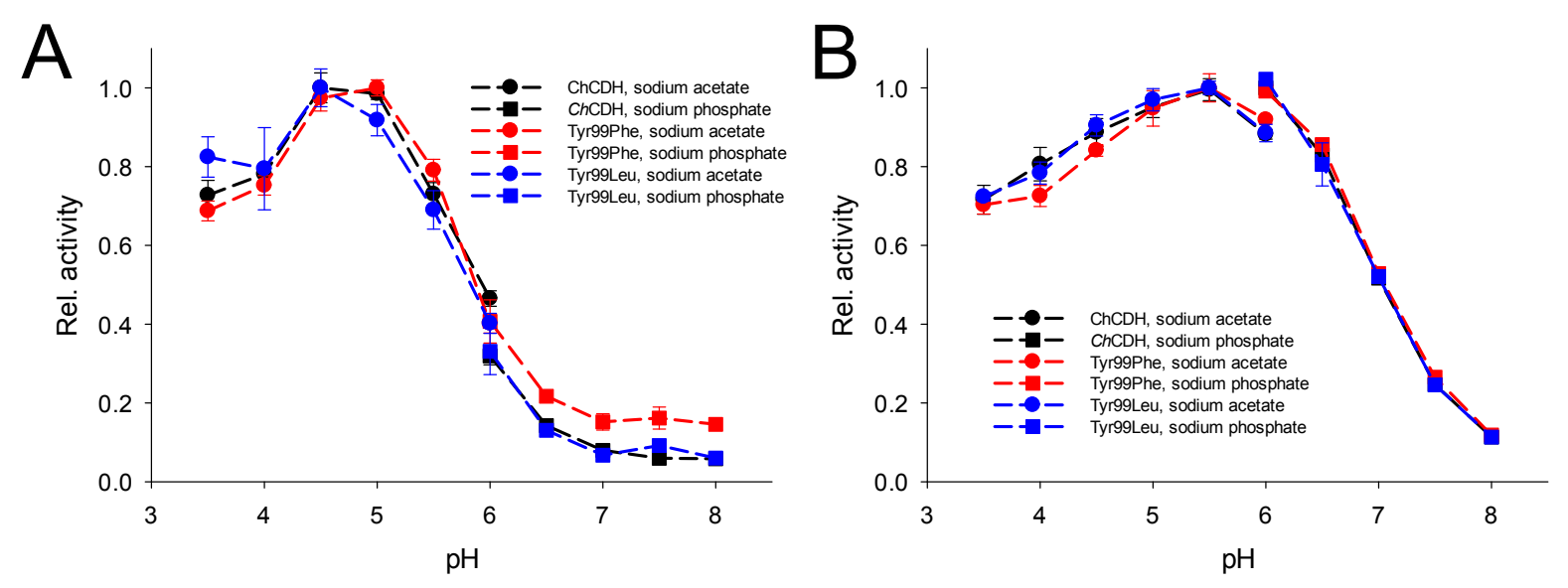

Figure S6. pH-dependent steady-state activities of ChCDH, Tyr99Phe and Tyr99Leu. $A$, pH-dependent activities of $C h \mathrm{CDH}$ variants using cytochrome $c$ as the terminal electron acceptor, which is exclusively reduced by the CYT-domain of CDH. Assays contained $20 \mu \mathrm{M}$ cytochrome $c$ and $10 \mathrm{mM}$ cellobiose as saturating substrate. $\boldsymbol{B}, \mathrm{pH}$-dependent activities with dichlorophenol-indophenol (DCIP), a FAD specific electron acceptor. Assays contained 300 $\mu \mathrm{M}$ DCIP and $10 \mathrm{mM}$ cellobiose. All assays were carried out at $30^{\circ} \mathrm{C}$. Error bars show \pm S.D. $(n=3)$ 

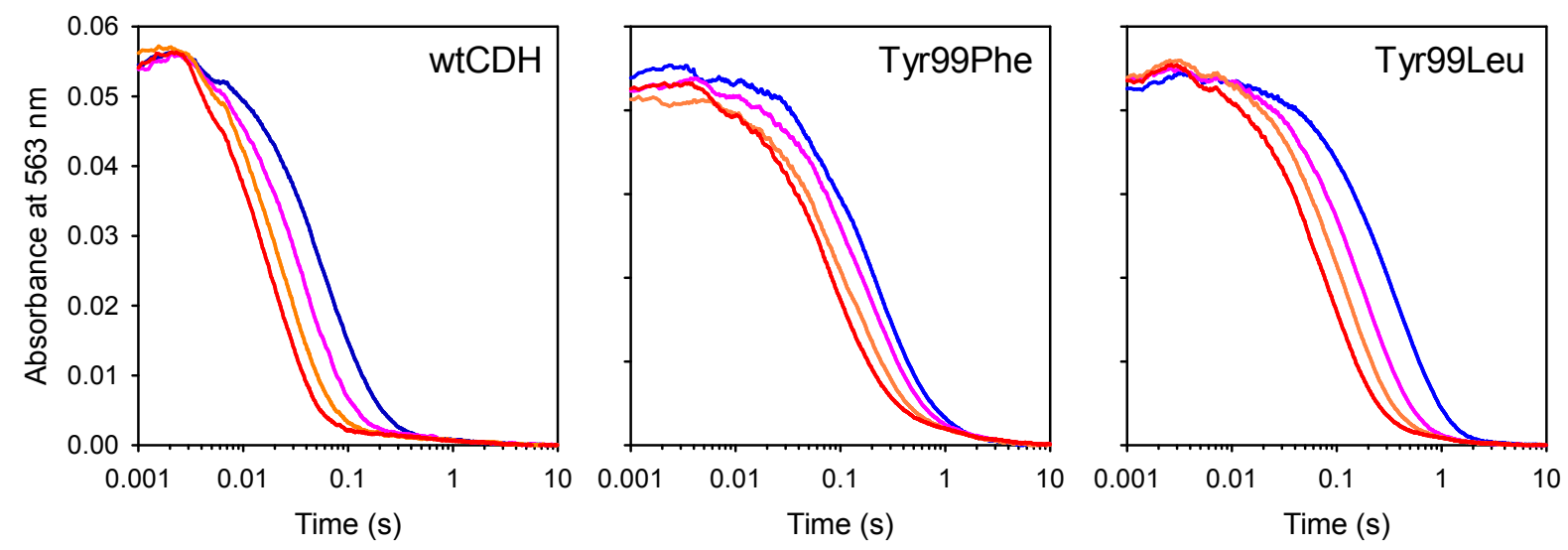

Figure S7. Interprotein electron transfer between CDH and LPMO. Wild type and variant CDHs $(2.5 \mu \mathrm{M})$ were stoichiometrically reduced with cellobiose in an anaerobic glove box and mixed with increasing concentrations of $N c$ LPMO9C (blue: $12.5 \mu \mathrm{M}$, purple: $25 \mu \mathrm{M}$, orange: $37.5 \mu \mathrm{M}$, red: $50 \mu \mathrm{M}$ ) at $30^{\circ} \mathrm{C}$. Traces are the average of three independent repeats. All concentrations are those after mixing. 


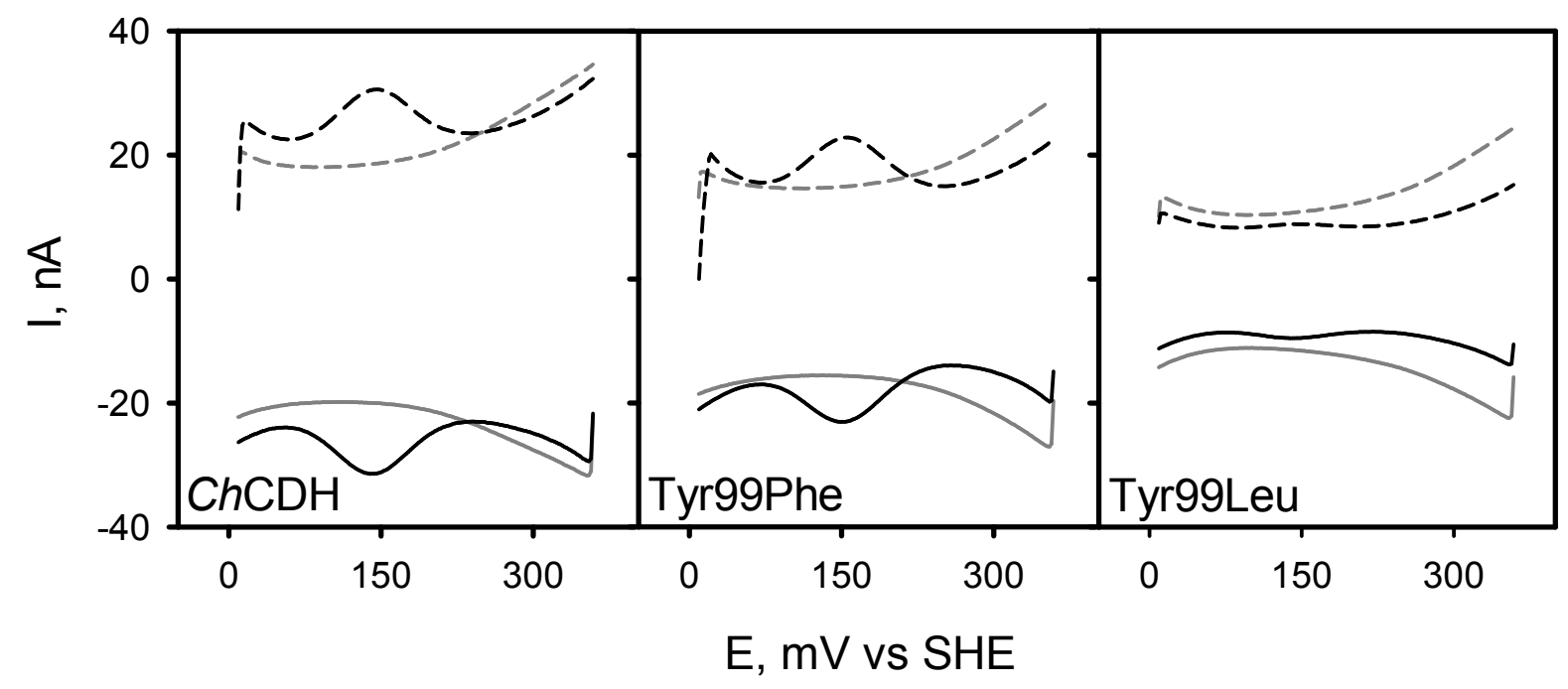

Figure S8. Square wave voltammograms of CDH variants. Enzymes were immobilized on

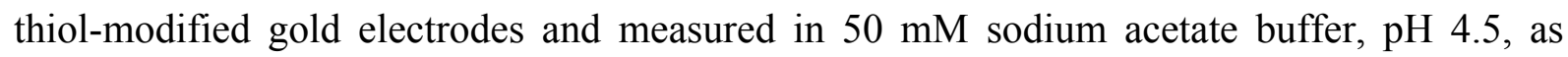
described in the Materials and Methods section of the article. Forward scans are shown as dashed black lines, reverse scans are indicated by black lines. Blank scans in the absence of $\mathrm{CDH}$ are shown as grey dashed lines (forwards scans) or grey lines (reverse scans). 
(a)

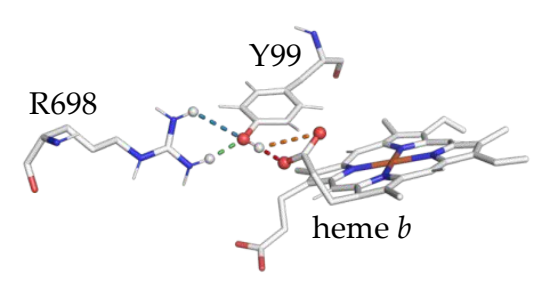

(b)

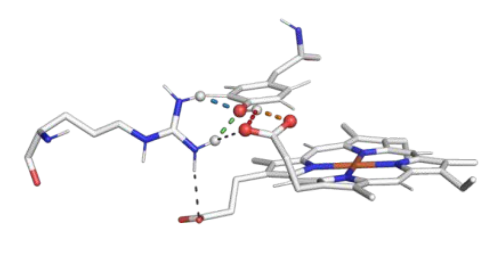

(c)

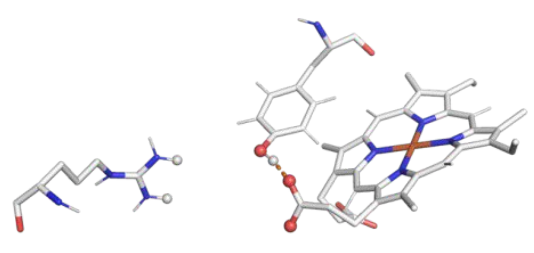

Figure S9. Three clusters from a $50 \mathrm{~ns}$ simulation of $C h C D H$ PDB ID: 4QI6). RMSD-based clustering was performed on the 3D coordinates of Tyr99, Arg698 and heme $b$ with a $2 \AA$ cutoff. H-bonds of Tyr99 with Arg698 and/or heme $b$ are highlighted with dashes (a). H-bonds have been calculated by considering the oxygen and hydrogen atoms of the Tyr99 hydroxyl group as acceptor and donor, respectively. Four possible H-bonds have been found and are shown as dashes. Atoms involved in these H-bonds are shown as spheres. The color of the dashes is mirroring the occurrence of the H-bonds during the simulation where red is $78.2 \%$, orange 47.4\%, green $42.6 \%$ and blue $22.8 \%$. Black dashes in (b) and (c) are indicating additional Hbonds between the three residues. Clusters and H-bonds have been calculated with the rmsdmat, cluster, postcluster and hbond programs as implemented in GROMOS++. ${ }^{2}$ 


\section{References}

(1) Webb, J. N.; Webb, S. D.; Cleland, J. L.; Carpenter, J. F.; Randolph, T. W. Partial Molar Volume, Surface Area, and Hydration Changes for Equilibrium Unfolding and Formation of Aggregation Transition State: High-Pressure and Cosolute Studies on Recombinant Human IFN-Gamma. Proc. Natl. Acad. Sci. U. S. A. 2001, 98, 7259-7264.

(2) Eichenberger, A. P.; Allison, J. R.; Dolenc, J.; Geerke, D. P.; Horta, B. A. C.; Meier, K.; Oostenbrink, C.; Schmid, N.; Steiner, D.; Wang, D.; van Gunsteren, W. F. GROMOS++ Software for the Analysis of Biomolecular Simulation Trajectories. J. Chem. Theory Comput. 2011, 7, 3379-3390 